Revista de Investigación en Psicología. Vol. 2, № 1. pp. 103 - 114

\title{
PSICOFISIOLOGÍA DE LA VIOLENCIA ASPECTOS DE ETOLOGÍA Y SOCIEDAD
}

\section{Edmundo Beteta Pacheco}

Con el objetivo investigar los factores biológicos en el desarrollo de la violencia, se presentan los estudios neurobiológicos de la agresión y la violencia en animales y en hombres. Se discute los aspectos controvertidos de la etología y la antropología social, se trata de interpretar la violencia social sobre la base de los factores psicobiológicos que aparentemente han sido relegados en los trabajos acerca de la violencia desarrollados en nuestro medio.

Palabras clave: violencia, agresión, etología, antropología social.

In order to study the biological factors of violence, it is presented the neurobiological research of agression in man and animals. We discuss the controversial aspects of the ethology and the social anthropology. Al the end, the interpretation of social violence should be done taking on account the psychobiological factors. Unfortunately the research about violence in Peru, it did not look for the biological aspects of this problem.

Key words: Violence, agression, ethology, social anthropology. 
Las bases experimentales, que demuestran la relación entre estructuras del cerebro y el comportamiento emocional, han sido estudiadas y publicadas en los últimos 60 años. Ellas constituyen el aporte de la Psicofisiología o Neurofisiología, disciplinas que representan las bases biológicas de la conducta (Beteta, 1992).

La violencia, una forma anormal de la expresión emocional, ha sido también estudiada experimentalmente en Psicofisiología. Estas investigaciones han servido como base a trabajos neurobiológicos, clínicos neurológicos y neuropsiquiátricos, en largas series de homicidas, violadores y pacientes portadores de diversos tipos de violencia (Mark \& Ervin, 1970).

La violencia y sus diversas formas de expresión constituyen un problema de salud pública que implica entre otras soluciones: reforma de la Educación, mejora de la calidad de vida, reforzamiento del denominado "corset cultural", estudios de despistaje neurológico y neuropsicológicos en niños y adolescentes, etc.

En nuestro medio las investigaciones publicadas están referidas fundamentalmente a los aspectos psicosociales, sin haber profundizado en las bases biológicas,(APEP, 1989; Delgado, 1989. Pimentel, 1987).

\section{ESTUDIOS NEUROBIOLÓGICOS}

La agresión es una tendencia instintiva destructiva, que requiere control; forma parte de muchas especies que la han requerido, en el sentido darwiniano, para sobrevivir el tiempo suficiente y reproducirse (proceso denominado por Spencer "la selección natural y la sobrevivencia del más eficiente"), sin embargo, estas especies, han desarrollado mecanismos de "control social", para evitar la agresión intra-específica y el aniquilamiento total, además, existe la posibilidad de que los elementos de la vida social de los animales dependan filogenéticamente de esta misma agresividad "controlada". Los etólogos dividen, desde este punto de vista, a los animales en predatorios (cazadores, armados de garras y colmillos, especializados en la defensa y el ataque) y suplicantes (cazados, poseedores de una rapidez y de una ligereza excelente, y por tanto, especializados en la huida). De este modo, frente a un peligro o una 
agresión, se mantiene el pattern de reacción biológica, del ataque asociado a la cólera, al furor o a la violencia, to fight, o la de la huida, asociada al temor o al miedo, to flight.

\section{Agresión en animales}

La agresión es entonces, un instrumento fundamental en la organización de la vida social de muchas especies. La conducta agresiva representa presión de supervivencia y selección, se trata de un Quantum de energía, dirigido hacia la destructividad y forma parte del equipamiento genético con diferentes formas de expresión; sin embargo la agresividad, de acuerdo a los etólogos, debe tener un control estructural ejercido por las características de la organización social de la especie, incluyendo los sistemas de dominio y territorialidad, así como, un control social, que se desarrolla por las características del comportamiento social de la especie, en el cual, la ritualización, en la técnica o proceso de lucha, determina que una disputa se resuelva sin violencia mediante gestos "ritualizados" de amenaza y sumisión (Scott, 1958). No existe, sin embargo, una separación crítica entre combate "ritualizado" y otro "real". Este mismo control social es la base de la "hermandad" animal, en donde existe el equilibrio real del poder con sus riesgos y limitaciones la interrelación social constituye un sistema total. Múltiples ejemplos ilustran la teoría presentada por los etólogos. Los lobos realizan una caza cooperativa con una forma suave de dominio que no excluye los machos jóvenes. Las colonias de babuinos y macacos, consiguen gracias a un aprendizaje social, un equilibrio de fuerzas que les brinda el control social (Aronson, 1972). Leyhausen, (1965) muestra la organización "política" de un vecindario de gatos: se mantienen a una distancia prudencial marcando ciertos hitos en su camino y todos los machos fuertes y saludables tienen oportunidad de reproducción; el macho de "mayor rango" pierde la superioridad de provocar a otro de "menor rango" porque tendría que luchar con el "inferior".

\section{La agresión en el hombre}

Teóricamente y siguiendo el marco de la evolución, la especie humana 
representa la racionalidad en el plano individual y la cultura en el ámbito social humano, pero no sólo está determinada por la razón y la tradición, sino que todavía está vinculada a las leyes del comportamiento instintivo, filogenéticamente adaptado. El impulso agresivo desarrolló por presiones de selección intraespecífica, creando las "virtudes guerreras", como valores culturales en su formación original, representando el móvil principal de la acción humana en la sociedad contemporánea. Si la agresividad es un fenómeno innato o una conducta aprendida, es una polémica que persiste hace muchos años, entre Psicólogos, Fisiólogos, Etólogos y Filósofos. En el concepto rousseauniano del buen salvaje, el hombre en su estado natural es una criatura feliz y buena, mientras otros. han adoptado el criterio de que el hombre es un "bruto" y sólo por medio de la ley y el orden social es posible doblegarlo; en fin, otros pensadores afirman que el hombre no sólo es un animal propenso a matar sino que su feroz destructividad es única entre los animales, sugiriendo que llamar brutal a la conducta del hombre es difamar a las otras especies.

No existen pruebas fisiológicas definitivas para concluir que la agresividad sea necesariamente instintiva aunque parece haberse demostrado que no necesita ser aprendida. De acuerdo a Berkowitz, en los humanos, gracias al aprendizaje, la agresividad es función de un juego complejo de propensiones innatas y respuestas aprendidas, por lo tanto, los patrones innatos de la conducta humana son "infinitamente modificables y flexibles". La agresión en los seres humanos es un modelo de construcción teórica diferente a la agresión de los animales. No son conceptos similares, "agresión", "lucha", "matar" y "guerra". Recordemos simplemente que existen formas sutiles de agresión verbal y simbólica, y que toda violencia no es agresiva si recordamos por ejemplo la actitud predatoria de los cazadores o el acto de matar de los Konyac Nagas, que lograban resultados específicos (actos violentos) sin animadversíon, furor u odio contra la víctima. Las propensiones naturales del hombre de acuerdo al criterio enunciado, no llegan a ser responsables de las matanzas sistemáticas por que la programación genética del hombre está ligada a una cultura y una tecnología, de otro modo no sería necesario el adoctrinamiento para preparar a ciertos grupos para la lucha. Justamente el desarrollo de las armas -reemplazó de colmillos y garras- está destinado a evitar la 
inhibición específica de matar. Por eso, de acuerdo a Lorenz (1986), el hombre es un omnívoro inofensivo que no tiene armas naturales ni mecanismos de seguridad, creados por la filogenia, los cuales impiden a los "carnívoros profesionales" aplicar indebidamente su poder para matar a los animales de su propia especie. En la evolución humana no existe el mecanismo defensivo de control social que impida atacar a su enemigo a pesar de los actos de humildad de la víctima para aplacar al agresor. La agresión no es una mera respuesta a la frustración, es más bien un impulso universal que debe ser diferenciado de la hostilidad de grupo en el cual existe un "entusiasmo militante" conectado a todas las formas de objetivos políticos.

\section{Aspectos de antropología social y etología}

En la terminología actual de Antropología Social y Etología algunas dicotomías conceptuales clásicas se fusionaron, tales como instinto/cultura, biología/sociedad, tradicional/occidental, salvaje/doméstico, hecho individual/hecho social; por eso la idea de racionalidad no ha reducido el nivel de organización social ni la comparación entre animales y hombre, pudiendo aceptarse la continuidad de las condiciones sociales de los grupos animales y humanos. Así Geertz (1964) señala que el Horno Sapiens aparece genéticamente encerrado entre las ropas de la cultura, planteamiento que Morris -en El mono desnudo-, trata de quitársela, sin mayor éxito. Es importante destacar, que mediante los mecanismos de herencia de conducta, los individuos están dotados de pautas o diseños de motivación y acción que precedieron a las Instituciones sociales, por esto, de acuerdo a Halsey, los sociólogos generalmente han presentado cierta hostilidad hacia las teorías biológicas de la sociedad (Gardiner, 1970). No parece existir razón, lógica o heurística para tratar a las ciencias sociales y a las biológicas como si fueran campos separados. La acción social humana "es el resultado de un prolongado proceso de selección natural", afirman Tiger y Fax (1966), y por lo tanto, los "hechos sociales" en el sentido de Durkheim (1938), no son los únicos "hechos sociales". Se trata, de acuerdo con Freeman (1966) que tanto en los casos humanos como en casos animales existen otros "hechos sociales" o "formas de base filogenética de 
adaptación social, que determinan al final en forma decisiva y de modo variable el curso del comportamiento social y la naturaleza de las relaciones sociales". El estudio científico de las adaptaciones culturales incluye, obligatoriamente, el estudio de la naturaleza impulsiva del hombre, la cual es parte integrante de la adaptación.

Las técnicas etológicas han sido utilizadas con cierto éxito en la observación del comportamiento social de infantes y pacientes esquizofrénicos, relacionando sus trabajos con la psicología social aunque no necesariamente toda la vida social se exprese en términos de conducta social. Igualmente, en primatología se pueden hacer comparaciones directas en el nivel sociológico entre el material animal y el humano, tratando de demostrar que ciertos rasgos de la sociedad humana pudieron evolucionar a partir de la organización social básica y de las condiciones de vida de los primeros primates y pre-homínidos (Grant, 1965).

En los párrafos anteriores, a propósito de la agresión en animales y el hombre, habíamos adelantado el término de control social referido al control de acción individual que debe cumplir funciones sociales para el grupo también identificado como legislación primitiva. Para Nadel (1955), existe autorregulación referida al comportamiento tradicional, que requiere pocos controles y el control social, que actúa cuando el primero se debilita. Todos los problemas sociales resultan entonces problemas de control social, se define el control social como la totalidad de las pautas culturales por lo cual la sociedad, cada grupo e individuo, vencen tensiones y conflictos por medio de equilibrios temporales que obligan a realizar nuevos esfuerzos creadores, es decir, el control social es todo o nada, pero a nuestro juicio, es necesario destacar que la naturaleza innata del hombre es la materia prima del control social (Nadel, 1955).

Tratando de establecer los vínculos funcionales entre vida social animal y humana, deben buscarse los instintos comunes, diseñados para lograr el control social. Nos referimos a las intersecciones entre dominio y territorialidad en las sociedades animales, y dominio y autoridad en las sociedades humanas. Está más o menos demostrado que la organización territorial reduce el contacto agresivo. Refiriéndose, a grupo o población como una colección grande de individuos con límites más o menos permanentes, Reynolds (1965), basado en el desarrollo de sistemas 
sociales jerárquicos en Primates en cautiverio y del hombre primitivo, en comunidades, establece que ambos tratan de controlar la frecuencia y la intensidad de los contactos para neutralizar los efectos "cara a cara" que ocurren en un ambiente superpoblado. Leyhausen (1965) demostró que los gatos "se vuelven más dominantes cuando están confinados y amontonados". De acuerdo a Darwin, el freno primario al continuo aumento de los hombres es la dificultad de ganarse la subsistencia y de vivir con comodidad, así, desde las sociedades más primitivas, se observó que ellas trataron de alcanzar la dignidad humana. Wynne (1962) define la sociedad "como una organización, capaz de proporcionar competencia de tipo tradicional"; el mismo autor piensa que la restricción del tamaño de la población en los grupos humanos se realiza mediante mecanismos sociales, en los cuales, los factores limitantes no son "el pan y la manteca sino los ostíones y el Champagne", refiriéndose a las dificultades para alcanzar una posición de prestigio en la sociedad.

Ciertos rasgos de la vida social humana son sobrevivientes de un antiguo conjunto de sistemas dispersores. Los animales y el hombre primitivo tuvieron sistemas autorreguladores del control de la población que no existió en los pueblos civilizados. De acuerdo a Russell y Russell (1968), el aumento de la presión de la población de animales y humanos tiene una respuesta predecible: desorganización y tensión social que culmina con el debilitamiento de la población y probablemente una "victimación selectiva" de mujeres y niños, proceso considerado un "mecanismo homeostático" de control.

Terminaremos diciendo que la pérdida de control social, tanto en la sociedad animal como en la humana ocurre como resultado de un quebrantamiento de la estructura, en algún nivel, de la micro o macro organización. El control social no puede ser definido en términos exclusivamente antropológicos por que los controles institucionales del comportamiento representan las partes más importantes de un complejo continuo de procesos, están estructurados en todos y cada uno de los puntos que uno quisiera analizar. Estas complejas interrelaciones no se detienen frente a la naturaleza humana; indiferenciada y engañosa, sino que forman una continuidad con la organización social que compartimos con otras especies. 


\section{HACIA UNA INTERPRETACIÓN DE LA VIOLENCIA SOCIAL}

Con los aportes de la Etología y la Antropología Social presentados, podemos empezar reconociendo que el hombre es la criatura mejor dotada en el Universo, lo cual no significa discriminación para el resto de las especies, sino al contrario, la elevación de las especies hasta las diferentes formas de inteligencia. Esta concepción no descarta que estemos insertados en el proceso biológico de la evolución y que tanto el hombre como la sociedad comparten muchas características con el animal y su sociedad. El meollo del problema no es necesariamente social sino en todo caso psicobiológico, por lo cual debe destacarse la personalidad como un grupo de características que incluyen la tradición, la escala de valores y otras variantes dinámicas de interacción entre la planificación genética cerebral y el medio social; por tanto no se debe caer en el desliz de referir la conducta humana sólo en términos de influencia social. En cuanto a la conducta humana agresiva, ya se ha discutido que el ser humano tiene disposiciones e impulsos agresivos, pero de ningún modo es un animal predatorio para su propia especie. La agresión en la escala humana está estructurada a despecho de su racionalidad, en el comportamiento instintivo como un impulso universal que es indispensable en la selección natural, en la adaptación cultural y en la dinámica del control social.

La conducta agresiva está genéticamente vinculada a la cultura y la tecnología y por ende normada por las Instituciones Sociales. Es evidente que para interpretar la violencia en una sociedad debemos de buscar las causas en el denominado control social que actúa, como se ha mencionado, cuando se pierde la autorregulación. En ese control se encuentra la estructura cultural, por la cual, todos los grupos e individuos logran dominar los conflictos mediante esfuerzos creativos para conseguir lo que podría denominarse "homeostasis social" cuando ésta se ha perdido. El dominio y la autoridad tendrá que restaurarse en los controles institucionales, investigando qué nivel de la organización es responsable, sin olvidar que el factor decisivo está en la naturaleza intrínseca del hombre.

Se ha referido también, que la agresividad humana puede alcanzar una "feroz agresividad", pero, que todos los individuos normales tienen una 
inhibición específica de matar y que las propensiones naturales de violencia nunca son responsables de los asesinatos sistemáticos, los cuales representan acciones de individuos con disturbios psicopatológicos, o de grupos que han desarrollado un aprendizaje de hostilidad generalmente asociado a objetivos políticos.

En fin, se ha determinado que el control social se pierde cuando los mecanismos de control de la población son precarios como resultado de una superpoblación, y cuando la competencia se hace muy complicada para que los individuos desarrollen sus capacidades y logren un determinado nivel social de acuerdo con sus aspiraciones y realización personal Es evidente que siempre existe una solución para estos problemas; aunque los mecanismos sean complejos y los resultados se logren a largo plazo, los individuos utilizarán el máximo de creatividad para llegar probablemente a soluciones transitorias. Esta situación se observa en sociedades como la capitalista y la antigua comunista que tienen un sistema de control social que para muchos estudiosos de las ciencias sociales favorece la violencia institucionalizada. De otro lado, en las sociedades sin desarrollo la depravación social por falta de medios e incentivos educacionales para que los individuos consigan sus objetivos lleva inevitablemente a un modelo anómalo de las relaciones entre los hombres, determinando diversas formas de conducta agresiva y violencia.

En muchos países de diferente desarrollo cultural desde hace varios años, en relación a la quiebra del control social, han proliferado grupos extremistas que, con diferentes propuestas políticas, han desarrollado actos de violencia causando la muerte de muchas personas. Un periodista de Le Monde en 1970 señalaba "cuántos muertos más faltan para restablecer el equilibrio en una sociedad enferma". Desde esa fecha, se estudió la prevalencia de los muertos en diferentes países: 3.5 por 100.000 habitantes en USA, 0.4 en el Japón y 0.15 en Gran Bretaña y aunque en el Perú, no se ha realizado puntualmente este estudio, se conoce que desde 1970, han muerto 22,000 personas por acciones de terrorismo.

Otro indicativo del estado de violencia en la sociedad es el tráfico de armas, constituyendo estos elementos, como se ha mencionado, los artefactos que bloquean la inhibición natural y específica de matar, facilitando y potenciando el impulso agresivo en sujetos predispuestos por 
un defecto psíquico o una personalidad proclive a ser adoctrinada para quebrar el orden social e individual, llegando fácilmente a la destrucción y al aniquilamiento. Un informe de 1970, en USA reveló que los ciudadanos civiles en Chicago poseían 358,000 armas, cantidad que de acuerdo al General G. Kanez, puede equipar 20 divisiones del Ejército de USA.

La literatura nacional revisada enfrenta la violencia, como violencia social; y en su definición de "violencia estructural" no existen parámetros de distinción biológicos, antropológicos y/o neuropsicológicos. Existe una coincidente explicación final, para diferentes actos de violencia, denominada (bajo el concepto de anomía) "específica" incluyendo los actos efectuados por el terrorismo, que se atribuyen a nuestro deterioro económico, social, cultural. En su conjunto, constituyen el mecanismo esencial de la violencia social, estructural o específica, para citar diferentes términos que asocian factores similares.

En cierto modo, entonces, se tendrían que justificar, el pensamiento de algunos etólogos quienes afirman que las sociedades animales están mejor organizadas que las humanas. Es difícil, por eso, seguir manteniendo la hipótesis básica de la violencia referida en términos de frustraciones, porque la solución de todos los problemas sociales debe requerir probablemente tanto tiempo como el empleado en el proceso de desarrollo de los mismos problemas.

La confrontación de diferentes criterios, tomando como esencia al hombre mismo, se hace necesaria para explicar la violencia. No son suficientes la pobreza ni el deterioro socio-cultural, tal vez la marginación social sea el caldo de cultivo para que desarrollen y proliferen ciertos individuos portadores de síndromes neuropsiquiátricos, quienes de acuerdo a sus frustraciones esenciales psicosociales han elaborado un marco conceptual doctrinario, aparentemente con objetivos políticos, basado en el resentimiento social, el odio, la destrucción y el sadismo. Se trata de un tipo de violencia irracional y fanática que es capaz de establecer y producir una reacción en cadena -como la "replicación viral"- en otras personalidades predispuestas, desarrollándose así "una cultura fanática al servicio de la muerte".

Al final de esta revisión debe considerarse que los aspectos neurológicos y neurobiológicos son de gran importancia para tener un 
amplio marco conceptual de la violencia. No existen, tal vez, factores biológicos independientes para explicar muchas formas de violencia. Igualmente, los factores sociales son predisponentes pero no causales de la violencia. Se requiere una conjunción de factores psicosociales y psicobiológicos que resultarían en las peores formas de violencia cuando el individuo es portador de un disturbio psíquico grave, un desorden psicopatológico, o tal vez una disfunción cerebral encubierta.

Por este motivo algunas formas terribles de violencia destructiva no se observan entre los animales. Se requiere el ingrediente de anormalidad psicobiológica que no ha sido suficientemente destacado en los trabajos nacionales sobre violencia.

Recordemos que Mussolini distinguió "la violencia que libera y la violencia que esclaviza; una moral y otra inmoral" y también en esa época, Hitler escribió: "lo esencial para el éxito, es un perpetuo empleo constante y regular de la violencia". Es evidente que Mussolini y Hitler son arquetipos de personalidades anormales, responsables de una gran cantidad de muertos. De acuerdo con Schlesinger (1969) el recurrir a la violencia implica el fracaso de la razón, sólo se justifica cuando todos los medios han sido agotados, y el uso de la fuerza queda como el último camino para alcanzar fines racionales.

Cualquier tipo de violencia, expresada en guerras y terrorismo, ha tenido una duración limitada. Siguiendo el pensamiento de Howard Munford Jones: "la celebración de la violencia terminará con la misma, cualquiera que sean los cambios políticos o sociales, a menos que toda la historia esté equivocada y se ponga de acuerdo en la destrucción del hombre y la cultura" (Schelesinger, 1969).

\section{REFERENCIAS BIBLIOGRÁFICAS}

APEP (1989). Siete ensayos sobre la violencia en el Perú. Lima: F.C.E.

Aronson, E. (1972). The social animal. San Francisco: W.H. Freeman Co.

Beteta, E. (1992). Neurobiología de la violencia. Boletín de la Sociedad Peruana de

Neurología. 3(3): 116-120.

Delgado, C. (1989). Terrorismo y estado. en Estado y sociedad en el Perú. Lima: N. Strotmann, Graf. San Pablo. 
Durkheim, E. (1938). Rules of sociological method. Londres: Free Press,. Freeman, D. (1966). Social anthropology and the scientific study of human behaviour. Man NSI 330-342.

Gardiner, W. (1970). Psychology. A story of a search. California: Wadsworth Pub. Co.: Belmont.

Geertz, C. (1964). The transition to humanity. en Fax. Horizonts of anthropology, Allen and UNWIN, Londres, 37 - 49.

Grant, E.C. (1965). An ethological description of some schizophrenic patterns of Behaviour. en Proceedings of the feeds symposium of behavioral disorders, New York.

Leyhausen P. (1965). Communal organization of solitary mammals. en Ellis, Ed.: Social organization of animal communities, Symposia of the zoological society of London, 14, 249 - 263.

Lorenz, K. (1986). Sobre la agresión: el pretendido mal. Buenos Aires: Siglo XXI. Mark, Y. \& Ervin, F. (1970). Violence and the brain. New York : Harper \& Row Pub. Inc.

Nadel, S. (1955). Foundations of social anthropology. Londres: Cohen and West.

Pimentel, C. (1987). Familia y violencia en la barriada. Lima: Los Pinos.

Reynolds, V. \& Reynolds, F. (1965). Chimpanzees of the budongo forest. En Devore,

Ed.: Primate behaviour. New York: Hold, Rinebart \& Winston:, 368 - 424.

Russell, C., Russell, W. (1968). Violence, monkeys and man. Londres: Mac. Millan.

Schelesinger, A. Jr (1969). The crisis of confidence. Ideas. Power and violence in America. Vol. 1, Boston: Houghton \& Miffin, Co:.

Scott, T. (1958). Agression in animals. University of Chicago Press: Chicago.

Tiger, L., Fox, R. (1966). The zoological perspective in social science. Man NSI. 75-8. Wynne - Edwards, V. (1962). Animal dispersion in relation to social Behaviour. Edinburgo: Oliver and Boayd. 